\title{
nature
}

24 May 2001 Volume 411 Issue no 6836

\section{A discipline buried by success}

Chemistry suffers from multiple image problems. Chemists working at its boundaries should acknowledge and celebrate their roots, while those at its core have much to celebrate too.

$\mathrm{C}$ hemistry's goal of understanding the form and function of molecules and studying how they interact is at the very heart of scientific endeavour. Yet its versatility comes at a price: recognition — or, more accurately, lack of it.

Just what is chemistry? The subject is no longer restricted to its academic organic, inorganic and physical divisions. In addition to well-known territories such as catalysis, organic synthesis, polymers and materials science, chemistry has extended to other less familiar areas - the use of small molecules in 'chemical genetics', screening drug candidates with combinatorial techniques and developing new analytical techniques that touch virtually every other part of science. The subject's sheer diversity - the American Chemical Society alone has over 30 divisions - makes generalizations about it impossible.

Perhaps such a definition is unnecessary. But lack of an accurate and identifiable chemistry 'brand' means that the discipline is easily misunderstood, and those working in it are frequently underappreciated. This is the basis of the discipline's infamous image problem. Chemists have allowed those from outside the field to characterize it - to define what chemistry is and what it is not.

To the public, chemical science is too often synonymous with the industry with which it shares its name. So chemistry means belching chimneys and poisoned rivers, not life-saving medicines and spaceage materials. To other researchers, policy-makers and, crucially, young scientists, it is sometimes seen as a mature discipline with its most important and stimulating work behind it. Chemists, on the other hand, tell a different story. They speak excitedly about the promise of molecular electronics, the challenges presented by the need for sustainable energy and the opportunities for bespoke pharmaceuticals presented by the human genome sequence.

\section{A nalys is in demand}

The subject's reputation is not a trivial issue. If chemistry is to achieve half as much in this century as it did in the last, it must fight to attract the brightest young scientists. And to do that, image is important. Furthermore, basic research in both academic and industrial chemistry needs a shot in the arm. Basic chemistry research in companies is suffering as research budgets are tightened. And basic chemistry research in universities is being overshadowed by the continued growth in support for biology. As one chemist laments: "biology is just a lot more attractive to people now."

But chemists and chemistry have never been more vital to science and society than now. As the borders between scientific disciplines blur (a process that will only continue), fundamental chemistry skills such as synthesis and analysis will be crucial for the interdisciplinary subjects that emerge. Indeed, chemists are already flocking in increasing numbers to the edges of their discipline to collaborate with biologists, physicists, engineers and computer scientists.

Research at these fringes is dynamic, fascinating and of great potential benefit. Chemists are vital to projects such as the use of medical research imaging to probe development and the study of molecular recognition and binding using synthetic cell membranes. Chemical analysis of protein folding and techniques based on mass spectroscopy are needed to capitalize on genomics, and it is chemical self-assembly techniques that are driving the nanotechnology bandwagon. This eye-catching interdisciplinary research offers chemistry a chance to polish its image. But first, chemists must learn to celebrate and promote their contributions.

As we discuss on page 408, one reason for chemistry's tarnished image is that rival fields have claimed many of its brightest moments. The reasons for this are subtle and numerous, but, as George Whitesides at Harvard University suggests, modesty could play a role. If that's true, chemists should stop hiding their Bunsen burners under bushels.

\section{Champions hip gaps}

Chemical societies are evolving to acknowledge the changing face of the subject they represent. In Germany, 2003 will be the year of chemistry, as the German Chemical Society publicly celebrates the subject's diversity. In Britain, the Royal Society of Chemistry is relaxing its membership criteria so that those doing chemistry, but who lack a classical chemistry background, can join. The American Chemical Society is trying to stamp chemistry's brand on emerging topics such as nanotechnology and proteomics through new divisions, journals and conferences.

These are welcome steps in the right direction. But if chemistry is to lay stronger claims to its future achievements, more of the frontline chemists streaming across the discipline's borders into attentiongrabbing multidisciplinary research must make their voices heard. They should proclaim their roles to colleagues and try to ensure that chemical contributions are made known to the media.

This may be difficult - the biological aspects of such projects are often easier for non-experts to understand. But the 1,000 chemists attending the recent meeting of the American Chemical Society in San Diego produced just a handful of press releases between them. If chemists want a better image they must lose their modesty.

Perhaps then, more of the chemical-sciences limelight will reflect back onto 'core' chemistry topics that also deserve greater attention. There are many. A recent poll of US chemists by the head of chemistry at the Massachusetts Institute of Technology, Stephen Lippard, produced a 'wish-list' of more than 20 exciting areas in basic chemistry that are ripe for discovery (see Nature 406, 807-808; 2000). These include self-replicating molecules, the search for reagents and pathways capable of breaking inert chemical bonds, designing customized catalysts for some reactions and eliminating the solvents from others. Such developments, and an improved yield, specificity and versatility of chemical reactions by greater control over the molecules involved, are, as Lippard says, driving a "quiet revolution" in chemistry.

But here, too, chemists should help their societies tell undergraduates, schools and the media what these breakthroughs could lead to. Tellingly, Lippard stops short of identifying potential applications of his wish-list to surrounding fields, and therefore their possible implications for the world at large. "We are confident our colleagues in those areas can make these connections," he says.

That is surely a missed opportunity. If more chemists established these connections themselves, and talked up the potential benefits, their contribution would not be so easily overlooked. Revolutions, after all, should be noisy. 